\title{
Corner overgrowth: Bending a high mobility two-dimensional electron system by $90^{\circ}$
}

\author{
M. Grayson ${ }^{(a)}$, D. Schuh, M. Huber, M. Bichler, and G. Abstreiter \\ Walter Schottky Institut, Technische Universität München, D-85748 Garching, Germany
}

(2 Nov 2004)

Introducing an epitaxial growth technique called corner overgrowth, we fabricate a quantum confinement structure consisting of a high-mobility GaAs/AlGaAs heterojunction overgrown on top of an ex-situ cleaved substrate corner. The resulting corner-junction quantum-well heterostructure effectively bends a two-dimensional electron system (2DES) at an atomically sharp $90^{\circ}$ angle. The high-mobility 2DES demonstrates fractional quantum Hall effect on both facets. Lossless edge-channel conduction over the corner confirms a continuum of $2 \mathrm{D}$ electrons across the junction, consistent with Schroedinger-Poisson calculations of the electron distribution. This growth technique differs distinctly from cleaved-edge overgrowth and enables a complementary class of new embedded quantum heterostructures.

The fabrication of quantum confined electron systems with increasingly complex geometries will become important as advances in technology reduce the size and dimensionality of electronic devices. In standard lowdimensional semiconductor systems, the confinement potential is atomically sharp only in the epitaxial growth direction and smooth on the order of several $100 \AA$ by gating or etching in the in-plane directions. One approach to achieve sharper in-plane confinement is to imbed quantum heterostructures within a crystal by using a sequence of orthogonal growths. Ground-breaking work using crystal regrowth techniques [1] and cleaved-edge overgrowth [2] utilized two distinct growth steps to produce high-mobility transport structures with sharp quantizing potentials in multiple directions $[1,3-10]$. In this Letter we introduce a new corner overgrowth technique that, in a single growth step, creates atomically sharp quantum confinement in two perpendicular directions which meet at a corner junction. We apply this technique to fabricate an L-shaped junction between two orthogonal high-mobility two-dimensional electron systems (2DES) and demonstrate lossless flow of quantum Hall edge channels across the junction as proof of the continuity of the $2 \mathrm{D}$ system.

We call our device the corner quantum-well heterojunction (CQW), fabricated by overgrowing a standard GaAs/AlGaAs heterojunction structure on an ex-situ cleaved corner. To achieve high-quality growth across the corner, both facets must have the same Miller index class. Since ex-situ cleaving is used to create the atomically sharp corner-substrate, we will restrict growths to two particular geometries that expose orthogonal $\{110\}$ - class planes. One can either cleave a (110)-GaAs substrate wafer once in the orthogonal (11) Type I), or one can take a (001)-GaAs wafer and cleave twice in the (110) and (110) planes (Fig. 1, Type II). In both cases, the shared corner between the (110) and (110) planes serves as the overgrowth corner and is mounted facing the molecular flux. For shorthand, we refer to (110) as the $s$-facet and (110) as the $p$-facet (corresponding to 'substrate' and 'precleave', respectively, for Type I samples).

The samples were grown in a Epi Gen-II molecular beam epitaxy (MBE) system with an ultra-high vacuum ambient $\left(P \approx 10^{-11}\right.$ mbar $)$. The $\hat{\mathbf{z}}$-axis in Fig. 1 is the rotation axis of the substrate as well as the axis around which the molecular sources are symmetrically arranged at an azimuthal angle $\theta_{\Sigma}=33^{\circ}$. The molecular flux $\Phi_{0}$ is first calibrated with RHEED on a standard flat substrate. The corner-substrate is then mounted in its place with both $s$ - and $p$-facets at a nominal $\theta_{s}=90^{\circ}-\theta_{p}=45^{\circ}$ angle. Under continuous rotation of angle $\alpha$ around the $\hat{\mathbf{z}}$ axis, the flux incident on the tilted $s$-facet will consist of an oscillating component which averages to zero, and a constant component equal to the RHEED calibrated flux $\Phi_{0}$ projected onto the tilted surface, $\Phi_{s}=\Phi_{0} \cos \left(\theta_{s}\right)$ [11]. The complementary $p$-facet will correspondingly see an average flux of $\Phi_{p}=\Phi_{0} \cos \left(\theta_{p}\right)=\Phi_{0} \sin \left(\theta_{s}\right)$. One main result of this work is that in spite of fluctuations in the molecular flux due to rotation, we nonetheless achieve high quality crystal growth on the tilted facets of the corner substrate. After oxide desorbtion, the sample is overgrown under rotation $(d \alpha / d t=7 \mathrm{rpm})$ at a substrate temperature of $T_{s u b}=460^{\circ} \mathrm{C}$ with all molecular fluxes increased by a factor of $1 / \cos \left(45^{\circ}\right)=\sqrt{2}$ to compensate for the geometrically reduced flux on the tilted substrate. For a typical growth, the beam equivalent pressure $\mathrm{BEP}_{\mathrm{As}_{4}}=5 \times 10^{-5} \mathrm{mbar}$, and $\mathrm{BEP}_{\mathrm{Ga}}=$ $2.2 \times 10^{-7}$ mbar, corresponding to a RHEED calibrated Ga growth rate of $2.4 \AA /$ s on a flat substrate.

In the SEM picture in Fig 1. we confirm the sharpness of the corner growth morphology in a test cornerovergrown superlattice structure. Using the RHEED calibrated flux values, the three dark bands on the left $p-$ (right $s-$ ) side of the sample are $810 \AA$ (560 $\AA$ ) AlAs separated by lighter GaAs/AlGaAs layers of varying thickness $1620 \AA, 1620 \AA$ and $2430 \AA$ (1120 , $1120 \AA$, and $1680 \AA)$ from the surface downwards. The corner junction clearly maintains a sharp $90^{\circ}$ corner profile even under regrowth. The slightly thicker layers on the left result from a growth angle of $\theta_{s}=56^{\circ}$ with the ratio of thicknesses $d$ on the two sides equal to $d_{s} / d_{p}=\tan \left(\theta_{s}\right)$. 
The jagged cleave is a consequence of forcing a break in a non-cleavage (001) plane, perpendicular to the two natural cleave planes (110) and (1 $\overline{1} 0)$.

The transport structure that is the focus of this paper is a CQW heterojunction, analogous to a single heterointerface 2DEG in planar structures. The growth consists of an AlGaAs/GaAs superlattice buffer and a base layer of GaAs, followed by growth on each facet of nominally $1200 \AA \quad \mathrm{Al}_{0.3} \mathrm{Ga}_{0.7} \mathrm{As}$, Si- $\delta$ doping, $3000 \AA \quad \mathrm{Al}_{0.3} \mathrm{Ga}_{0.7} \mathrm{As}$, and a $100 \AA$ GaAs cap layer (see Fig. 2 inset). The sample is electrically contacted with indium, alloyed onto the sample edges away from the overgrown corner. By measuring the 4-point longitudinal resistance of the two facets at $350 \mathrm{mK}$ (Fig. 2), the densities of the two facets can be independently measured with the result: $n_{s}=$ $1.07 \times 10^{11} \mathrm{~cm}^{-2}$ and $n_{p}=1.30 \times 10^{11} \mathrm{~cm}^{-2}$. Observation of fractional quantum Hall effect minima forming at this strength at filling factor $\nu=2 / 3$ at $350 \mathrm{mK}$ attests that both facets have a transport mobility of order $\mu \approx 5 \times$ $10^{5} \mathrm{~cm}^{2} / \mathrm{Vs}$.

We explain the dissimilarity in the two densities by considering that the thickness of growth on the two facets will not be equal if the corner is not mounted at exactly $45^{\circ}$ during growth. Assuming that the dopant density is on the verge of parallel conduction at $\theta_{s}=45^{\circ}$ as per design, the curve calculated in Fig. 3 shows the $2 \mathrm{D}$ density $n_{s}\left(\theta_{s}\right)=n_{p}\left(90^{\circ}-\theta_{s}\right)$ as well as the parallel conduction density of the $\delta$-doping layer $n_{\delta}$ as a function of substrate tilt angle $\theta_{s}$. One sees the predominant effect of large tilt angles $\left(\theta_{s}>45^{\circ}\right)$ is thinner layers, and a corresponding rapid depletion of the $2 \mathrm{D}$ density due to the proximity of the surface pinning potential. Conversely, with smaller tilt angles $\left(\theta_{s}<45^{\circ}\right)$ the layers are thicker, and the density drops only slightly due to the increasing spacer thickness $d$ between the 2D and the saturated dopant layer. With decreasing $\theta_{s}$, the excess doping rapidly accumulates electron density in the parallel conducting dopant layer. A growth angle of $\theta_{s}=47^{\circ}$ would account for the appropriate ratio of $n_{s} / n_{p}$ observed in the measured device.

To demonstrate continuity of the $2 \mathrm{D}$ electron system, we first measure the 2-point resistance across the corner at zero $B$. The cross-corner resistance is $\sim 1 \mathrm{k} \Omega$ comparable to the 2 -point resistivities within a given facet, our first indication of a continuous $2 \mathrm{D}$ system. For a more rigorous demonstration of $2 \mathrm{D}$ continuity, we apply a magnetic field and tilt the sample to an angle where the filling factors on the two facets make simple integer ratios. Fig. 4 shows the case $\nu_{s} / \nu_{p}=2\left(\theta_{s}=31.3^{\circ}\right)$, and the inset shows the edge-state diagram for the $\nu_{s}: \nu_{p}=2: 1$ case. With a current supplied from contact $\mathrm{Y}$ to contact $\mathrm{Z}$ the 4-point resistance across the corner between voltage contacts $\mathrm{A}$ and $\mathrm{B}, R_{A B}^{c c}$, is zero $(0 \pm 0.5 \Omega)$ whenever both $2 \mathrm{D}$ systems are gapped (dashed line, $\nu_{s}: \nu_{p}=2: 1,4: 2$, $6: 3)$. From the Landauer-Buttiker diagram, this means the outermost edge channel is transmitted across the corner with no backscattering, demonstrating the continuity of the $2 \mathrm{D}$ electron system across the corner. For voltage contacts C-D in the same gapped regions, the LandauerButtiker equations predict the observed $R_{C D}^{c c}=\frac{\nu_{s}-\nu_{p}}{\nu_{s} \nu_{p}} \frac{h}{e^{2}}$.

We calculated the self-consistent Hartree electron density at the CQW heterojunction, revealing an enhanced density exactly at the corner. Integrating the electron density cross-sectionally in the (100) direction and projecting onto the corner profile indicated by the dashed line in the inset of Fig. 3, we arrive at a measure of the local 2D density along the CQW, unfolded and plotted as a solid line in Fig. 3, inset. We observe a single, deeply bound 1D-wire ground state at the corner $(d=0)$ with calculated ground energy $E_{F}-E_{0}=7 \mathrm{meV}$ and spatial extent $\Delta x_{0}=200 \AA$ (dotted line). All other states have much weaker binding energy $E_{F}-E_{n \geq 1} \leq 4 \mathrm{meV}$ and much larger spatial extent $\Delta x_{n \geq 1}>1000 \AA$, so we subtract out the $1 \mathrm{D}$ density and label the remainder as two-dimensional in character (dashed line). Breaking the total charge density into 2D and 1D densities, we observe in the Fig. 2 inset, that the $2 \mathrm{D}$ states maintain a remarkably uniform density $n=1.2 \times 10^{11} \mathrm{~cm}^{-2}$ up to within $\lambda_{F} / 2$ of the corner, where $\lambda_{F} / 2=\sqrt{\pi / 2 n}=360 \AA$ is half the Fermi wavelength. We also see small Friedel oscillations away from the corner with the expected period $\lambda_{F} / 2$. We note that the calculated $2 \mathrm{D}$ continuum of electrons across the corner is consistent with the observed low cross-corner resistance and lossless edge channel conduction.

In conclusion, we have created a new kind of quantum confinement structure by overgrowing an ex-situ cleaved corner substrate. Self-consistent Hartree calculations predict a tightly bound wire state at the junction, along with a continuum of $2 \mathrm{D}$ electrons which approach the corner with a remarkably uniform density. The bent $2 \mathrm{D}$ electron system shows high mobility and continuity as characterized with magnetotransport.

This work was supported in part by the DFG Schwerpunktprogramm Quanten-Hall-Systeme (SFB 348). M.G. thanks the A.v. Humboldt Foundation for support, J. Smet and K. von Klitzing for stimulating discussion, and S. F. Roth for SEM pictures.

(a) Email: matthew.grayson@wsi.tum.de

[1] A. Zaslavsky, D. C. Tsui, M. Santos, and M. Shayegan, Appl. Phys. Lett. 58, 1440 (1991).

[2] L. N. Pfeiffer, K. W. West, H. L. Stormer, J. P. Eisenstein, K. W. Baldwin, D. Gershoni, and J. Spector Appl. Phys. Lett. 56, 1697 (1990).

[3] A. Yacoby, H. L. Stormer, K. W. Baldwin, L. N. Pfeiffer, and K. W. West, Solid State Commun. 10177 (1997).

[4] M. Grayson, C. Kurdak, D. C. Tsui, S. Parihar, S. Lyon, and M. Shayegan, Sol. State Elec. 40, 233 (1996).

[5] A. M. Chang, L. N. Pfeiffer, and K. W. West, Phys. Rev. Lett. 772538 (1996). 
[6] W. Kang, H. L. Stormer, L. N. Pfeiffer, K. W. Baldwin, and K. W. West, Nature 403, 59 (2000).

[7] A. Majumdar, L. P. Rokhinson, D. C. Tsui, L. N. Pfeiffer, and K. W. West, Appl. Phys. Lett. 763600 (2000).

[8] R. A. Deutschmann, W. Wegscheider, M. Rother, M. Bichler, G. Abstreiter, C. Albrecht, and J. H. Smet Phys. Rev. Lett. 861857 (2001).

[9] M. Huber, M. Grayson, M. Rother, W. Wegscheider, and G. Abstreiter, Physica E 12125 (2002).

[10] O. M. Auslaender, A. Yacoby, R. de Piccioto, K. W. Baldwin, L. N. Pfeiffer, and K. W. West Science 295 825 (2002).

[11] This simplified picture is valid as long as $\theta_{s}, \theta_{p}<90^{\circ}-\theta_{\Sigma}$.

FIG. 1. (Top) Cleaved corner substrates: Type I from (110) wafers, Type II from (001) wafers. (Bottom) SEM micrograph of corner overgrowth test structure. (Right) Schematic of growth geometry: rotation axis $\hat{\mathbf{z}}$, source angle $\theta_{\Sigma}$, substrate angle $\theta_{s}$, and precleave angle $\theta_{p}$.

FIG. 2. $R_{x x}$ for the two facets, with densities $n_{s}=1.07 \times 10^{11} \mathrm{~cm}^{-2}, n_{p}=1.30 \times 10^{11} \mathrm{~cm}^{-2}$. Fractional QH minima at $\nu=2 / 3$ attest to sample quality. Inset: Bent heterojunction quantum well structure.

FIG. 3. Calculated 2D $s$-facet carrier density $n_{s}$ and parallel conduction carrier density in $\delta$-dopant layer $n_{\delta}$ plotted as a function of substrate growth angle $\theta_{s}\left(\theta_{p}=90^{\circ}-\theta_{s}\right)$. Inset: Hartree calculation of charge density (solid), separated into 1D (dotted) and 2D (dashed) contributions.

FIG. 4. 4-point cross-corner resistances. $R_{A B}^{c c}=0$ and $R_{C D}^{c c}=\frac{\nu_{s}-\nu_{p}}{\nu_{s} \nu_{p}} \frac{h}{e^{2}}$ when both facets are gapped, as predicted by the Landauer-Buttiker formalism. (inset). 


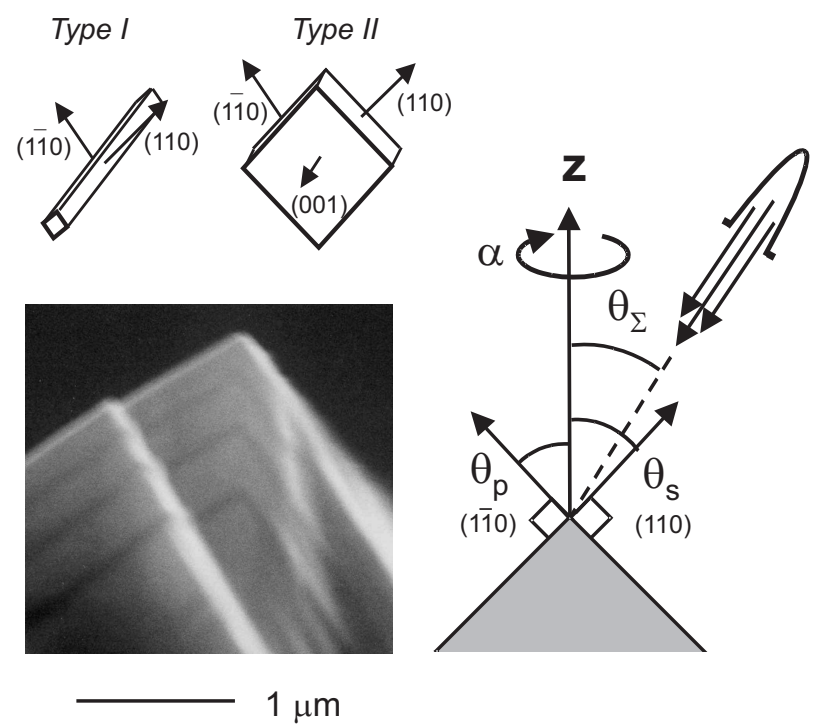

Fig. 1 


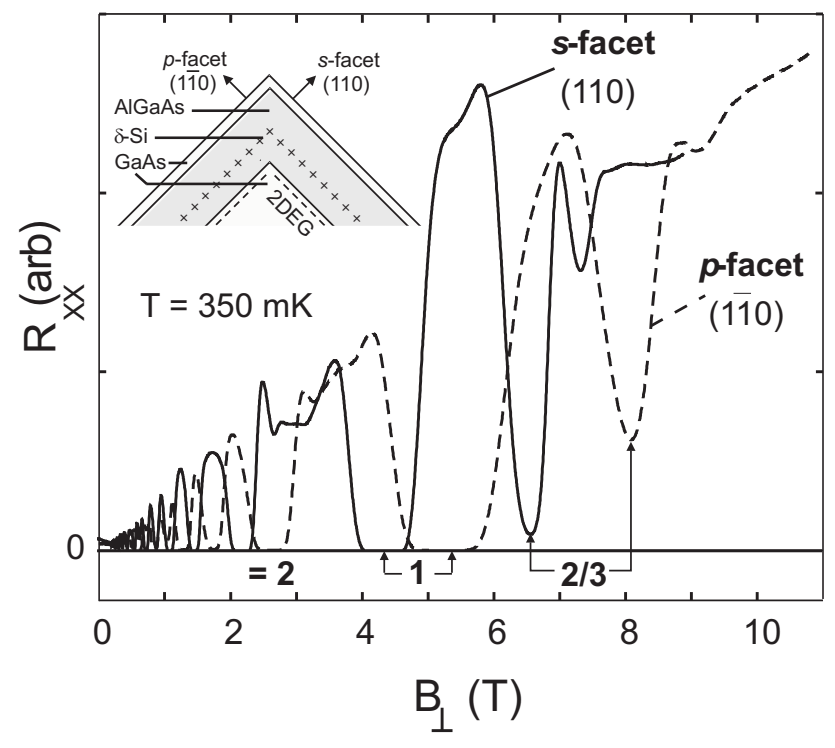

Fig. 2 